\title{
Gyroscopically Stabilized Oscillators and Heat Baths
}

\author{
Anthony M. Bloch, ${ }^{1}$ Patrick Hagerty, ${ }^{1}$ Alberto G. Rojo, ${ }^{2}$ and \\ Michael I. Weinstein ${ }^{3}$
}

Received March 14, 2003; accepted November 19, 2003

\begin{abstract}
In this paper we analyze the stability of a gyroscopic oscillator interacting with a finite- and infinite-dimensional heat bath in both the classical and quantum cases. We consider a finite gyroscopic oscillator model of a particle on a rotating disc and a particle in a magnetic field and we examine stability before and after coupling to a heat bath. The heat bath is modelled in the finite-dimensional setting by a system of independent oscillators with mass. It is shown that if the oscillator is gyroscopically stable, coupling to a sufficiently massive heat bath induces instability even in the finite-dimensional setting. The key mechanism for instability in this paper is thus not induced by damping. The meaning of these ideas in the quantum context is discussed. The model extends the exact diagonalization analysis of an oscillator and field of Ford, Lewis, and O'Connell to the gyroscopic setting. We also discuss the interesting role that damping of Landau type plays in the infinite limit.
\end{abstract}

KEY WORDS: Oscillators; stability; gyroscopic forces.

\section{INTRODUCTION}

In this paper we investigate the stability properties of a gyroscopically stabilized system interacting with a finite or infinite-dimensional heat bath. As a standard model for a gyroscopic system, we consider a particle on a rotating disc and a charged oscillator in a magnetic field.

A gyroscopically stabilized system is one that is unstable without gyroscopic forces but becomes stable with the addition of these forces.

\footnotetext{
${ }^{1}$ Department of Mathematics, University of Michigan, Ann Arbor, Michigan 48109; e-mail: abloch@math.lsa.umich.edu and hagerty@umich.edu

${ }^{2}$ Department of Physics, Oakland University, Rochester, Michigan 48309; e-mail: rojo@ oakland.edu

${ }^{3}$ Department of Applied Physics and Applied Mathematics, Columbia University, New York, New York 10027, and Fundamental Mathematics Research Department, Bell Laboratories, 600 Mountain Avenue, Murray Hill, New Jersey 07974; e-mail: miw@research.bell-labs.com
} 
Infinitesimal dissipative perturbations are known to induce instability in Hamiltonian systems that are gyroscopically stabilized; see ref. 5.

Since the origins of dissipation (e.g., friction, viscosity,...) lie in the transfer of energy from one form (energy of one subsystem) to another form (that of a second subsystem) of a larger conservative system, it is natural to expect the analogue of the above destabilization phenomenon to be present within the more fundamental context of conservative systems which exhibit internal energy transfer. In refs. 12 and 13, we explore this in the context of a gyroscopic oscillating mechanical system coupled to an extended wave system (infinite string). Due to the coupling, motion within the mechanical system generates waves which can be carried off to infinity. Such radiation damping has been studied in models arising in the theory of quantum resonances, ionization type problems and nonlinear waves; for more detail see refs. 29-31, ref. 18, and references therein. There are many interesting papers of course discussing the role of dissipation in quantum systems and systems of quantum oscillators, for example, refs. 1, 7, 9, 10, $15,16,22,24$, and 33. We cannot begin to give a complete list here (and indeed our focus here is the classical rather than quantum setting) but refer the reader to the literature for other aspects of this problem.

We consider here two models of gyroscopically stabilized systems (a particle stabilized either by rotation or a magnetic field). We show that coupling to a finite (i.e., nonthermal) system of oscillators with sufficient coupling strength induces instability. This result is quite striking in the sense that one does not expect a finite reservoir to mimic dissipation and indeed the key mechanism for instability in this paper in not dissipation. We also however consider the infinite-dimensional limit. In this case dissipation does play a role which we discuss. A graphical criterion is given for determining the onset of instability.

We discuss briefly also the quantum setting where we show that while a stable oscillator has positive energy bound states, a gyroscopically stable oscillator has both positive and negative energy bound states. Coupling to the bath with sufficient coupling strength induces unbound states in the gyroscopically stable system.

Our analysis is for a model problem but there are interesting possible applications to systems with similar geometry such as Penning traps which are used to trap charged particles and obtain extremely precise measurements of atomic quantities. ${ }^{(6)}$

Our analysis extends the heat bath analysis of ref. 11 to the gyroscopic setting. We show here that there is a beautiful extension of their graphical (intersection-theoretic) criterion for stability to a more complex class of curves. A brief earlier version of some of these ideas is discussed in ref. 4. 
In the final section of the paper we discuss gyroscopic oscillators coupled to an infinite heat bath modelled by a string. We discuss in some detail the infinite limit and show that this limit we get an additional mechanism for instability, which we elucidate in a manner analogous to the study of Landau damping. This is related to the work on radiation induced instability discussed in refs. 12 and 13. We also discuss other types of infinite-dimensional coupling.

\section{EXAMPLES OF CHETAEV SYSTEMS}

In this section we discuss two physical realizations of gyroscopic oscillators: firstly a planar oscillator on a rotating plate and secondly a planar oscillator in a constant magnetic field.

\section{Planar Oscillator on a Rotating Plate}

One physical model of a gyroscopic system is a planar oscillator on a plate rotating with angular velocity $\omega$ (see Fig. 1). We write the Lagrangian as the kinetic energy minus the potential energy,

$$
L=\frac{1}{2}\left((\dot{x}-\omega y)^{2}+(\dot{y}+\omega x)^{2}\right)-\frac{1}{2}\left(\alpha x^{2}+\beta y^{2}\right) .
$$

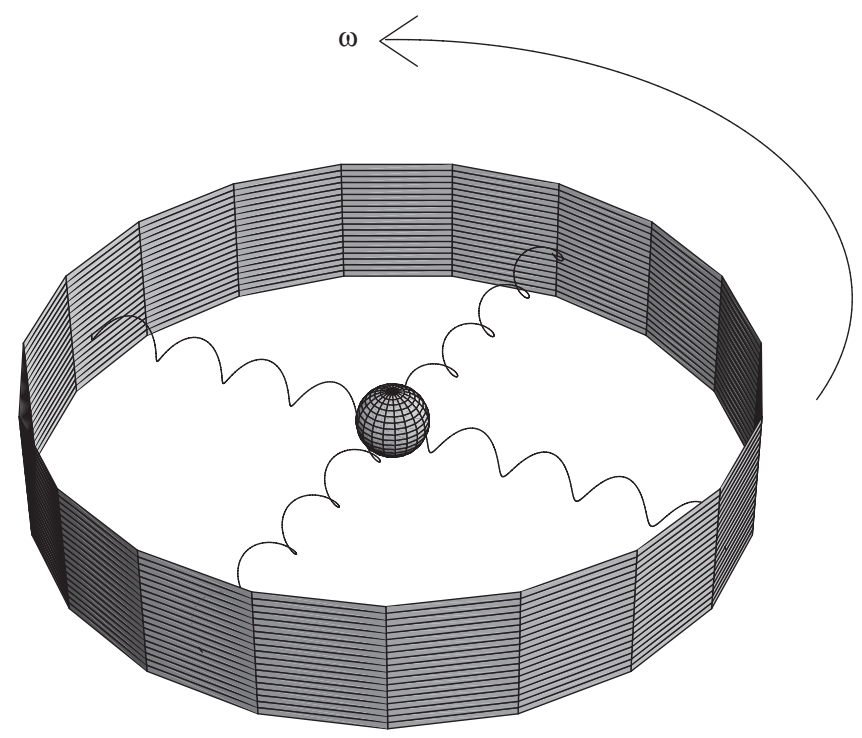

Fig. 1. Rotating plate with springs. 
In the Hamiltonian setting we have

$$
H=\frac{1}{2}\left(p_{x}^{2}+p_{y}^{2}\right)+\frac{1}{2}\left(\alpha x^{2}+\beta y^{2}\right)+H_{\omega},
$$

where

$$
H_{\omega}=\omega\left(p_{x} y-p_{y} x\right)
$$

and the associate momenta are given by

$$
\begin{aligned}
& p_{x}=\frac{\partial L}{\partial \dot{x}}=\dot{x}-\omega y \\
& p_{y}=\frac{\partial L}{\partial \dot{y}}=\dot{y}+\omega x .
\end{aligned}
$$

We obtain the equations of motion,

$$
\begin{aligned}
-\dot{p}_{x} & =\frac{\partial H}{\partial x}=\alpha x-\omega p_{y} \\
-\dot{p}_{y} & =\frac{\partial H}{\partial y}=\beta y+\omega p_{x} \\
\dot{x} & =\frac{\partial H}{\partial p_{x}}=p_{x}+\omega y \\
\dot{y} & =\frac{\partial H}{\partial p_{y}}=p_{y}-\omega x .
\end{aligned}
$$

Thus we have the following dynamics,

$$
\begin{aligned}
& \ddot{x}-2 \omega \dot{y}+\left(\alpha-\omega^{2}\right) x=0 \\
& \ddot{y}+2 \omega \dot{x}+\left(\beta-\omega^{2}\right) y=0 .
\end{aligned}
$$

We comment on this below.

\section{Planar Oscillator in a Constant Magnetic Field}

Another physical realization is the motion of a charged spherical pendulum in a magnetic field whose linearization is that of a charged planar oscillator in a magnetic field (see Fig. 2). We consider here the motion of such an oscillator (for details of the full nonlinear system see, e.g., ref. 13). 


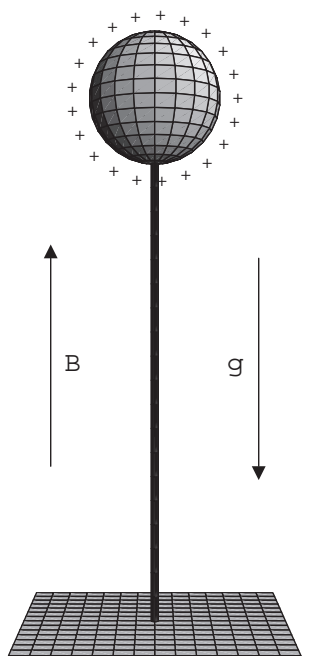

Fig. 2. Inverted spherical pendulum.

Let $\mathbf{B}$ be a divergence-free vector field. Let $\mathbf{A}$ be the vector potential, $\mathbf{B}=\nabla \times \mathbf{A}$. Note if we choose $\mathbf{B}$ to be the constant magnetic field in the direction normal to the plane of oscillation, the vector potential can be chosen as $\mathbf{A}=\frac{1}{2} \mathbf{B} \times \mathbf{q}$, where $\mathbf{q}=(x, y, 0)^{T}$ is the position of the oscillator.

Assume a normalization where the oscillator has unit mass and unit charge and that the speed of light is unity. The Lagrangian, $L: T \mathbb{R}^{2} \rightarrow \mathbb{R}$, is defined by:

$$
\begin{aligned}
L(\mathbf{q}, \dot{\mathbf{q}}) & =\frac{1}{2}\|\dot{\mathbf{q}}\|^{2}+\mathbf{A} \cdot \dot{\mathbf{q}}-U(\mathbf{q}) \\
& =\frac{1}{2}\left(\dot{x}^{2}+\dot{y}^{2}\right)+\mathbf{A} \cdot(x, y, 0)^{T}-\frac{1}{2}\left(\alpha x^{2}+\beta y^{2}\right) .
\end{aligned}
$$

Choosing $\mathbf{B}$ to be of constant strength $B$, normal to the plane of oscillation we have,

$$
L=\frac{1}{2}\left(\dot{x}^{2}+\dot{y}^{2}\right)-\frac{1}{2}\left(\alpha x^{2}+\beta y^{2}\right)+\frac{1}{2} B(x \dot{y}-y \dot{x}) .
$$

The associated Hamiltonian, $H: T^{*} \mathbb{R}^{2} \rightarrow \mathbb{R}$, is

$$
\begin{aligned}
H & =p_{x} \dot{x}+p_{y} \dot{y}-L \\
& =\frac{1}{2}\left(p_{x}^{2}+p_{y}^{2}\right)+\frac{1}{2}\left(\alpha x^{2}+\beta y^{2}\right)+H_{B},
\end{aligned}
$$

where

$$
H_{B}=\frac{1}{2} B\left(p_{x} y-p_{y} x\right)+\frac{1}{8} B^{2}\left(x^{2}+y^{2}\right),
$$


and the associated momenta are given by

$$
\begin{aligned}
& p_{x}=\frac{\partial L}{\partial \dot{x}}=\dot{x}-\frac{1}{2} B y \\
& p_{y}=\frac{\partial L}{\partial \dot{y}}=\dot{y}+\frac{1}{2} B x .
\end{aligned}
$$

In the above notation, $H_{B}$ is the contribution of a magnetic field.

We obtain the equations of motion,

$$
\begin{aligned}
-\dot{p}_{x} & =\frac{\partial H}{\partial x}=\left(\alpha+\frac{1}{4} B^{2}\right) x-\frac{1}{2} B p_{y} \\
-\dot{p}_{y} & =\frac{\partial H}{\partial y}=\left(\beta+\frac{1}{4} B^{2}\right) y+\frac{1}{2} B p_{x} \\
\dot{x} & =\frac{\partial H}{\partial p_{x}}=p_{x}+\frac{1}{2} B y \\
\dot{y} & =\frac{\partial H}{\partial p_{y}}=p_{y}-\frac{1}{2} B x .
\end{aligned}
$$

This gives the dynamics

$$
\begin{aligned}
& \ddot{x}-B \dot{y}+\alpha x=0 \\
& \ddot{y}+B \dot{x}+\beta y=0 .
\end{aligned}
$$

Remark 1. If $\alpha$ and $\beta$ are both negative, the oscillator in a field system is unstable for small $B$. However, if $B^{2}+\alpha+\beta>2 \sqrt{\alpha \beta}$, the oscillator stabilizes, i.e., the eigenvalues are on the imaginary axis - this is what is referred to as gyroscopic stabilization. For further details see below and ref. 5 .

Remark 2. The rotating disc affects the oscillator differently from a magnetic field - compare Eqs. (2.6) and (2.13). While the magnetic field only adds $\dot{q}$ terms, the rotating disc also adds additional $q$ terms to the dynamics. For Eqs. (2.13), we can see that in the case of a physically stable oscillator with $\alpha, \beta>0$ for rotation rate $\omega$ sufficiently large, the system becomes only gyroscopically stable, i.e., the coefficients $\alpha-\omega^{2}$ and $\beta-\omega^{2}$ are negative but the eigenvalues are on the imaginary axis due to the presence of gyroscopic terms. 
Remark 3. In either case above, if the system is gyroscopically stable it can be shown that adding a small amount of dissipation to the system renders it unstable (i.e., there are unstable eigenvalues). For a more precise statement and generalizations see Section 3.

\section{GYROSCOPIC SYSTEMS}

We recall here some general properties of linear systems with gyroscopic forces. The systems above are examples of such systems.

The general form of a gyroscopic system is

$$
M \ddot{\mathbf{q}}+S \dot{\mathbf{q}}+V \mathbf{q}=0,
$$

where $\mathbf{q} \in \mathbb{R}^{n}, M$ is a positive-definite symmetric $n \times n$ matrix, $S$ is skew, and $V$ is symmetric. As in ref. 5 we shall call this the Chetaev system (see ref. 8).

We say the system is gyroscopically stable if for $S=0$ the origin is an unstable equilibrium, but for $S \neq 0$, the origin is a spectrally stable equilibrium (i.e., the eigenvalues of the linearized system have non-positive real part). The matrix $S$ is sometimes referred to as a magnetic term which arises from charged oscillators in a magnetic field.

An important property of this system is that it is a normal form for a simple mechanical system about a relative equilibrium which is given modulo an abelian group. That is, it is the normal form for a system defined on the cotangent bundle $T Q$ of a configuration space $Q$ where the Lagrangian is given by kinetic minus potential energy. (We recall that a point in $T Q$ is a relative equilibrium with respect to the action of a group if the dynamical orbit through that point remains in the group orbit.) One can obtain a similar normal form in the case of a non-abelian group. (See refs. 5 and 27.) The magnetic term naturally arises in the symplectic form when investigating the quotient space. However, we can obtain the same dynamics from a canonical symplectic form and an augmented Hamiltonian. This can always be done by the momentum shifting lemma (see ref. 23).

If we include the magnetic terms in the symplectic form, the Hamiltonian, $H: T^{*} Q \rightarrow \mathbb{R}$, of a gyroscopic system is the sum of the kinetic energy and potential energy

$$
H=\frac{1}{2} \mathbf{p}^{T} M^{-1} \mathbf{p}+\frac{1}{2} \mathbf{q}^{T} V \mathbf{q} .
$$

The Poisson bracket is given by

$$
\{F, K\}_{\text {magnetic }}=\frac{\partial F}{\partial q^{i}} \frac{\partial K}{\partial p_{i}}-\frac{\partial K}{\partial q^{i}} \frac{\partial F}{\partial p_{i}}-S^{i j} \frac{\partial F}{\partial p_{i}} \frac{\partial K}{\partial p_{j}} .
$$


It also useful to write the system as a Hamiltonian system with respect to the canonical bracket.

A simple method of seeing this is a follows:

We wish to write the system (3.1) as a Hamiltonian system with canonical symplectic form

$$
\{F, K\}_{\text {canonical }}=\frac{\partial F}{\partial q^{i}} \frac{\partial K}{\partial p_{i}}-\frac{\partial K}{\partial q^{i}} \frac{\partial F}{\partial p_{i}},
$$

and Hamiltonian

$$
H_{\text {magnetic }}=\frac{1}{2} p^{T} M^{-1} p+\frac{1}{2} q^{T}(V+W) q+p^{T} X q .
$$

Equating the resulting equations of motion with Eqs. (3.1) we see that the skew matrix $X$ and the symmetric matrix $W$ are implicitly defined by

$$
\begin{aligned}
X M+M X & =-S \\
W & =-X M X .
\end{aligned}
$$

Solving Eq. (3.5) for $X$, we explicitly have a change of variable from a Hamiltonian system with magnetic terms in the symplectic form to a Hamiltonian system with canonical symplectic form. It can in fact easily be shown using the Fredholm Alternative (see ref. 13 for details), that Eq. (3.5) has a solution for $X$.

Classically, the two representations of the Chetaev systems are equally useful. However, the canonical bracket is preferable when quantizing the mechanical system. (See refs. 2, 5, and 8 for further physical discussions.)

\section{Stability of Chetaev Systems}

In the sequel we investigate the stability of perturbed Chetaev systems, but first we summarize the stability of unperturbed Chetaev systems. The stability of the Chetaev system depends both on the signature of the bilinear form associated with a quadratic Hamiltonian and also on the magnetic terms in the symplectic form. In particular, the magnetic terms can stabilize gyroscopic systems with negative eigenvalues of $V$.

As for gyroscopic stability, the number of negative eigenvalues of the quadratic form plays a crucial part as ref. 8 discusses and which we summarize in the following proposition:

Proposition 3.1. Consider the canonical gyroscopic system $M \ddot{\mathbf{q}}+$ $S \dot{\mathbf{q}}+V \mathbf{q}=0$, where $M$ is a symmetric positive definite matrix, $S$ is a skewsymmetric matrix, and $V$ is a symmetric matrix: 
- If $V$ has a odd number of negative eigenvalues (counting multiplicity) then the origin is an unstable equilibrium.

- If $V$ has an even number of negative eigenvalues (counting multiplicity), we can choose $S$ so that the origin is a spectrally stable equilibrium.

Proof. Consider the associated first order linear operator, $L$,

$$
L=\left[\begin{array}{cc}
0 & I \\
-M^{-1} V & -M^{-1} S
\end{array}\right] .
$$

Let $p(\lambda)$ be the characteristic polynomial of the matrix $L$ and let $\lambda_{1}, \ldots, \lambda_{n}$ be the eigenvalues of the matrix $V$.

$$
\begin{aligned}
p(0) & =\operatorname{det} L \\
& =(-1)^{n} \operatorname{det}\left(-M^{-1} V\right) \\
& =\operatorname{det}\left(M^{-1}\right) \prod_{i=1}^{n} \lambda_{i} .
\end{aligned}
$$

Suppose that $V$ has an odd number of negative eigenvalues. Due to the positive definiteness of $M$, we have $p(0)<0$. We will show that $p(\lambda)$ changes signs as $\lambda$ increases.

$$
\begin{aligned}
\lim _{\lambda \rightarrow \infty} p(\lambda) & =\lim _{\lambda \rightarrow \infty} \operatorname{det}(L-\lambda I) \\
& =\lim _{\lambda \rightarrow \infty}(-1)^{2 n} \lambda^{2 n}+O\left(\lambda^{2 n-1}\right) \\
& =\infty .
\end{aligned}
$$

Hence, if $V$ has an odd number of negative eigenvalues (counting multiplicity) then the characteristic polynomial of $L$ has a positive real root and the origin cannot be gyroscopically stabilized.

Now to prove the second item, suppose that $V$ has an even number of negative eigenvalues (counting multiplicity). Stabilizing a pair of negative eigen-directions at a time, gyroscopic stabilization for $n=2$ is possible as shown in the introductory examples of Section 2. This completes the proof.

Gyroscopically stable systems exhibit interesting instability when perturbed by dissipative forces. Suppose now that $V$ has at least one negative eigenvalue. A key result of ref. 5 is that adding small dissipation always yields instability. More precisely it is shown that 
Theorem 3.2. Under the above conditions, if we modify the general Chetaev system by adding a small Rayleigh dissipation term,

$$
M \ddot{q}+(S+\epsilon R) \dot{q}+V q=0
$$

for small $\epsilon>0$, where $R$ is symmetric and positive definite, then the perturbed linearized equations

$$
\dot{z}=L_{\epsilon} z
$$

where $z=(q, p)$ are spectrally unstable, i.e., at least one pair of eigenvalues of $L_{\epsilon}$ is in the right half plane.

This result builds on basic work of refs. 8, 14, and 32. We refer to this as dissipation induced instability. Some of the radiation induced instabilities that arise in the gyroscopic Lamb model are analogous to the dissipation induced instability. We see that the Hamiltonian of a gyroscopically stabilized Chetaev system is indefinite. In this case, Rayleigh dissipation decreases the value of the Hamiltonian, but this does not bound the motion of the Chetaev system. In particular, the Hamiltonian may decrease to zero, while the displacement and velocities grow exponentially.

\section{OSCILLATOR COUPLED TO A BATH}

We now consider the gyroscopic oscillator coupled to a bath of oscillators via an augmented Lagrangian or Hamiltonian,

$$
L=L_{0}+L_{\text {bath }}
$$

where

$$
\begin{aligned}
L_{0} & =\frac{1}{2}\left(\dot{x}^{2}+\dot{y}^{2}\right)-\frac{1}{2}\left(\alpha x^{2}+\beta y^{2}\right)+\frac{1}{2} B(x \dot{y}-y \dot{x}) \\
L_{\text {bath }} & =\sum_{j=1}^{N} \frac{1}{2} m_{j}\left(\dot{x}_{j}^{2}+\dot{y}_{j}^{2}\right)-\frac{1}{2} m_{j} \omega_{j}^{2}\left(\left(x_{j}-x\right)^{2}+\left(y_{j}-y\right)^{2}\right) .
\end{aligned}
$$

Similarly, the Hamiltonian can be augmented as,

$$
H=H_{0}+H_{\text {bath }}
$$

where

$$
\begin{aligned}
H_{0} & =\frac{1}{2}\left(p_{x}^{2}+p_{y}^{2}\right)+\frac{1}{2}\left(\alpha x^{2}+\beta y^{2}\right)+H_{B} \\
H_{\text {bath }} & =\sum_{j=1}^{N} \frac{1}{2} m_{j}\left(p_{x_{j}}^{2}+p_{y_{j}}^{2}\right)+\frac{1}{2} m_{j} \omega_{j}^{2}\left(\left(x_{j}-x\right)^{2}+\left(y_{j}-y\right)^{2}\right),
\end{aligned}
$$


where $H_{B}$ is given by Eq. (2.10) and $0<\omega_{1}<\cdots<\omega_{N}$. For convenience we define $\omega_{-i}=-\omega_{i}$. This model extends the model described by ref. 11 to the gyroscopic setting. As discussed in that paper this model provides a good physical realization of oscillator-bath coupling. For simplicity of the computations here we restrict ourselves to the case $\alpha=\beta$. Similar results hold in the general case. We also restrict ourselves to the generic situation (see the proof for comments on genericity) though again similar results hold in the nongeneric setting.

The theorem below in fact generalizes the results of ref. 11 in a rather beautiful fashion, leading to the study of a more complex intersection problem.

We can show:

Theorem 4.1. Consider the system with Lagrangian (4.1) where $\alpha=\beta$ and the equation

$$
\left(\omega \pm \frac{1}{2} B\right)^{2}-\left(\alpha+\frac{1}{4} B^{2}\right)=\sum_{j=1}^{N} m_{j} \frac{\omega_{j}^{2} \omega^{2}}{\omega^{2}-\omega_{j}^{2}} .
$$

Generically we have the following:

The oscillator is stable if there exists $4 N+4$ real frequencies $\omega=\Omega_{i}$, $i= \pm 1, \ldots, \pm(2 N+2)$ which are solutions to Eq. (4.7). Let $\Omega_{1}$ be the smallest positive solution and let $\Omega_{i}$ be increasing with respect to the index $i$.

More precisely we have

(i) In the case of instability of the oscillator $H_{0}\left(\alpha+\frac{1}{4} B^{2}<0\right)$, there are only $4 N$ real solutions. Additionally, there are 2 pairs of imaginary solutions corresponding to instability.

(ii) In the case of strong stability of $H_{0}(\alpha>0)$, there are $4 N+4$ real, normal modes maintaining stability via coupling.

(iii) In the case of gyroscopic stability of $H_{0}\left(\alpha+\frac{1}{4} B^{2}>0\right)$, there are two possibilities. The values of $m_{j}$ and $\omega_{j}$ determine which occurs:

(a) If $\Omega_{1}<\omega_{1}$, then there are $4 N+4$ real, normal modes (counting multiplicities) corresponding to stability.

(b) If $\Omega_{1}>\omega_{1}$, then there are $4 N$ real, normal modes and 2 pairs of imaginary mode corresponding instability in the coupling. This case occurs for large $m_{j}$. 
Proof. The equations of motion are

$$
\begin{aligned}
\ddot{x}-B \dot{y}+\alpha x & =\sum_{j=1}^{N} m_{j} \omega_{j}^{2}\left(x_{j}-x\right) \\
\ddot{y}+B \dot{x}+\beta y & =\sum_{j=1}^{N} m_{j} \omega_{j}^{2}\left(y_{j}-y\right) \\
\ddot{x}_{j}+\omega_{j}^{2} x_{j} & =\omega_{j}^{2} x \\
\ddot{y}_{j}+\omega_{j}^{2} y_{j} & =\omega_{j}^{2} y .
\end{aligned}
$$

We seek normal mode solutions of the form

$$
\begin{aligned}
x(t) & =X_{0}(\omega) e^{i \omega t} ; & y(t) & =Y_{0}(\omega) e^{i \omega t} \\
x_{j}(t) & =X_{j}(\omega) e^{i \omega t} ; & y_{j}(t) & =Y_{j}(\omega) e^{i \omega t} .
\end{aligned}
$$

The equations of motion for the normal modes become

$$
\begin{aligned}
\left(-\omega^{2}+\alpha\right) X_{0} & =i B \omega Y_{0}+\sum_{j=1}^{N} m_{j} \omega_{j}^{2}\left(X_{j}-X_{0}\right) \\
\left(-\omega^{2}+\beta\right) Y_{0} & =-i B \omega X_{0}+\sum_{j=1}^{N} m_{j} \omega_{j}^{2}\left(Y_{j}-Y_{0}\right) \\
\left(-\omega^{2}+\omega_{j}^{2}\right) X_{j} & =\omega_{j}^{2} X_{0} \\
\left(-\omega^{2}+\omega_{j}^{2}\right) Y_{j} & =\omega_{j}^{2} Y_{0} .
\end{aligned}
$$

Eliminating the bath coordinates, we have

$$
\begin{aligned}
& \left(-\omega^{2}+\alpha\right) X_{0}=i B \omega Y_{0}+\sum_{j=1}^{N} m_{j} \frac{\omega_{j}^{2} \omega^{2}}{\omega_{j}^{2}-\omega^{2}} X_{0} \\
& \left(-\omega^{2}+\beta\right) Y_{0}=-i B \omega X_{0}+\sum_{j=1}^{N} m_{j} \frac{\omega_{j}^{2} \omega^{2}}{\omega_{j}^{2}-\omega^{2}} Y_{0} .
\end{aligned}
$$

We wish to find nontrivial solution to the following linear equation

$$
\left[\begin{array}{cc}
\omega^{2}-\alpha+\sum_{j=1}^{N} m_{j} \frac{\omega_{j}^{2} \omega^{2}}{\omega_{j}^{2}-\omega^{2}} & i B \omega \\
-i B \omega & \omega^{2}-\beta+\sum_{j=1}^{N} m_{j} \frac{\omega_{j}^{2} \omega^{2}}{\omega_{j}^{2}-\omega^{2}}
\end{array}\right]\left[\begin{array}{c}
X_{0} \\
Y_{0}
\end{array}\right]=\left[\begin{array}{l}
0 \\
0
\end{array}\right] .
$$


Hence, we have nontrivial normal mode solutions at the zeros of the function

$$
f(\omega)=\left(\omega^{2}-\alpha+\sum_{j=1}^{N} m_{j} \frac{\omega_{j}^{2} \omega^{2}}{\omega_{j}^{2}-\omega^{2}}\right)\left(\omega^{2}-\beta+\sum_{j=1}^{N} m_{j} \frac{\omega_{j}^{2} \omega^{2}}{\omega_{j}^{2}-\omega^{2}}\right)-B^{2} \omega^{2} .
$$

In the case where $\alpha=\beta$, we have

$$
\left(\omega \pm \frac{1}{2} B\right)^{2}-\left(\alpha+\frac{1}{4} B^{2}\right)=\sum_{j=1}^{N} m_{j} \frac{\omega_{j}^{2} \omega^{2}}{\omega^{2}-\omega_{j}^{2}}
$$

Generically Eq. (4.22) has $4 N+4$ distinct complex solutions corresponding to intersections between the two parabolae on the left hand side and the curve on the right. The latter curve has (for $\omega_{j}$ distinct) $2 N+1$ branches, the branch through the origin intersecting each parabola twice and each other branch intersecting each parabola once. Thus we obtain the $4 N+4$ intersections generically. In the case $B=0$ this number is halved as the parabolae coincide. In addition it is possible for tangencies to occur.

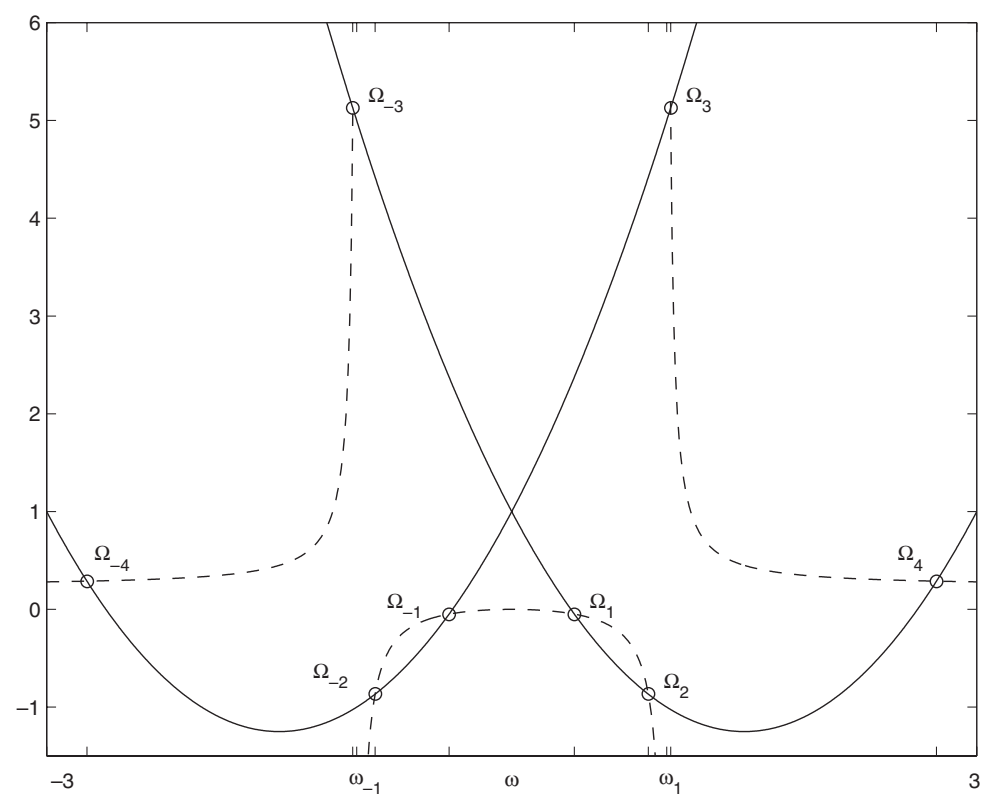

Fig. 3. Gyroscopic system coupled to a heat bath of one oscillator $(N=1)$ maintaining stability. Stable coupling with $\alpha=-1, B=3, m_{1}=\frac{1}{4}$, and $\omega_{1}=1$. Note $\Omega_{1}<\omega_{1}$ which implies that there are 8 real solutions. The dashed line is the graph of $\sum_{j=1}^{N} m_{j} \frac{\omega_{j}^{2} \omega^{2}}{\omega^{2}-\omega_{j}^{2}}$ while the solid lines are the graphs of the parabolas $\left(\omega \pm \frac{1}{2} B\right)^{2}-\left(\alpha+\frac{1}{4} B^{2}\right)$. 
Similar results follow in these nongeneric cases and we do not enumerate them here.

We can therefore see the oscillator is stable if there exist $4 N+4$ real frequencies which are solutions to the above Eq. (4.22). Complex solutions lead to instability.

The precise results now follow simply from examining the number of real intersections of the curves on the left and right sides of Eq. (4.22). For all cases it is helpful to refer to Figs. $3-6$ for the case $N=1$ and the case $N=2$.

(i) Follows since the parabolae in this case have no zeros.

(ii) Holds since $\alpha>0$ guarantees that (4.22) has a full set of solutions.

(iii) Follows from computing the minimum of $f(\omega)$ in the interval $\left(0, \omega_{1}\right)$. Each parabola intersects the graph of $\sum_{j=1}^{N} m_{j} \frac{\omega_{j}^{2} \omega^{2}}{\omega^{2}-\omega_{j}^{2}}$ between any successive singularities, $\left(\omega_{j}, \omega_{j+1}\right)$, for $j \geqslant 1$, accounting for $4 N$ real modes.

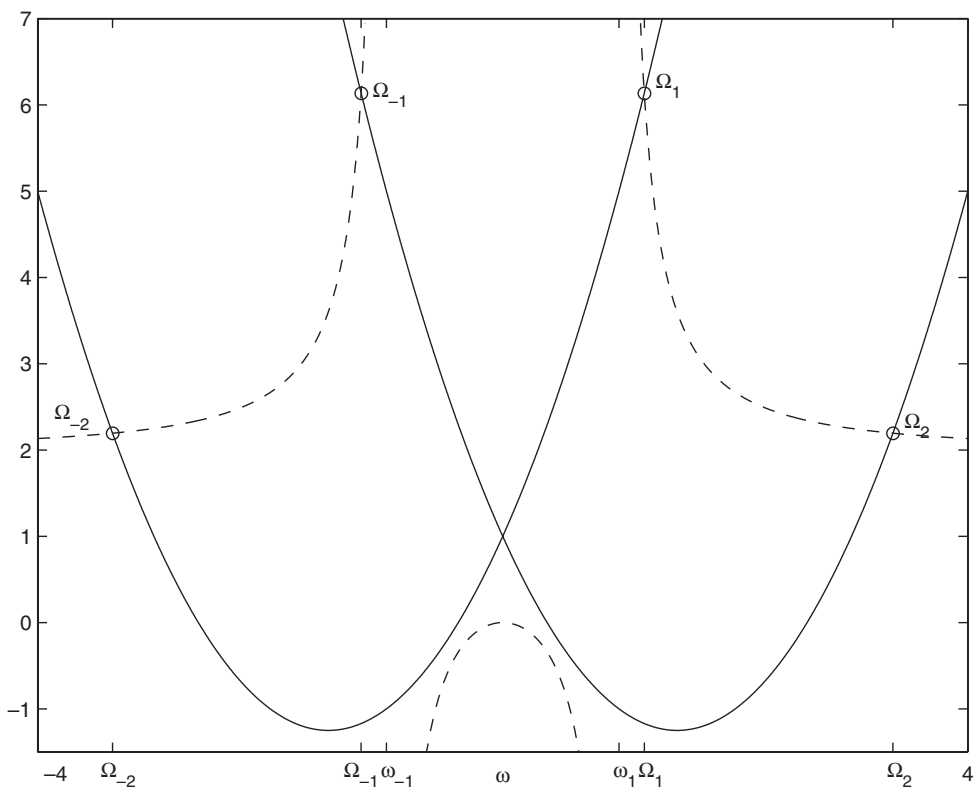

Fig. 4. Destabilization of a gyroscopic system coupled to a heat bath of one oscillator $(N=1)$. Unstable coupling with $\alpha=-1, B=3, m_{1}=2$, and $\omega_{1}=1$. Note $\Omega_{1}>\omega_{1}$ which implies that there are only 4 real solutions. The dashed line is the graph of $\sum_{j=1}^{N} m_{j} \frac{\omega_{j}^{2} \omega^{2}}{\omega^{2}-\omega_{j}^{2}}$ while the solid lines are the graphs of the parabolas $\left(\omega \pm \frac{1}{2} B\right)^{2}-\left(\alpha+\frac{1}{4} B^{2}\right)$. 


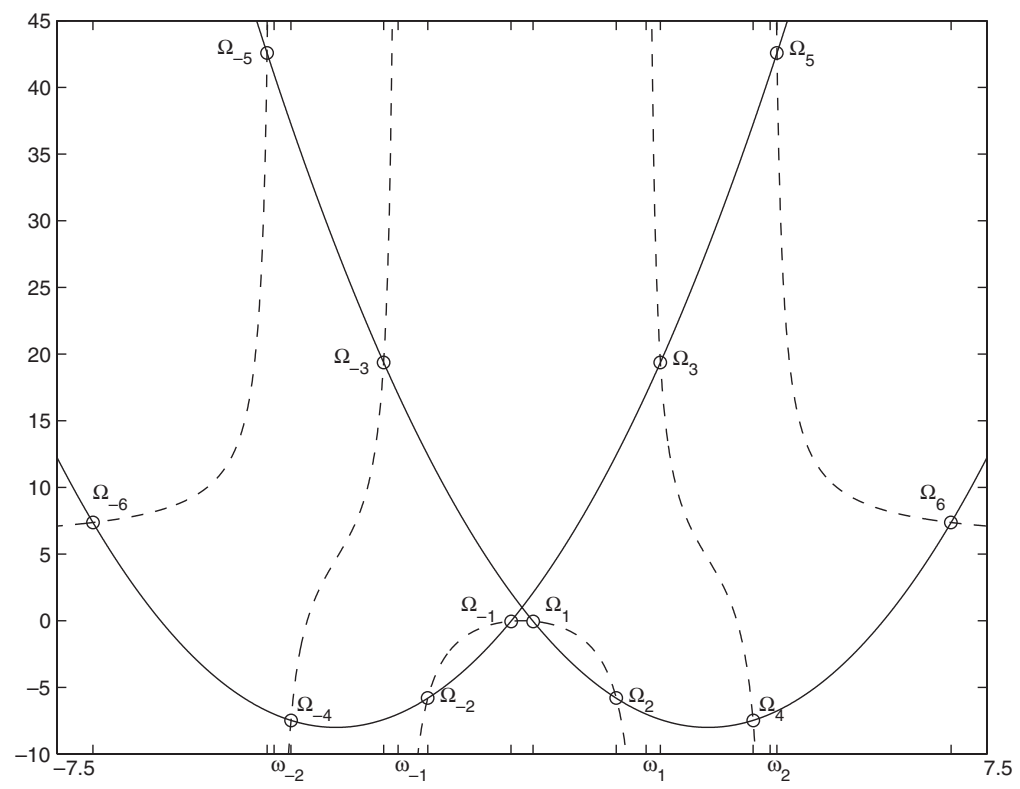

Fig. 5. Stable gyroscopic system coupled to a heat bath of two oscillators $(N=2)$. Stable coupling with $\alpha=-1, B=6, m_{1}=1, \omega_{1}=1, m_{2}=\frac{1}{8}$, and $\omega_{2}=4$. Notice the system has $4 N+4$ stable normal modes if $\Omega_{1}<\omega_{1}$. The dashed line is the graph of $\sum_{j=1}^{N} m_{j} \frac{\omega_{j}^{2} \omega^{2}}{\omega^{2}-\omega_{j}^{2}}$ while the solid lines are the graphs of the parabolas $\left(\omega \pm \frac{1}{2} B\right)^{2}-\left(\alpha+\frac{1}{4} B^{2}\right)$.

However it is the number of modes that exist in $\left[-\omega_{-1}, \omega_{1}\right]$ which determines stability. Counting multiplicities, there are $4 N+4$ real modes if $\Omega_{1}<\omega_{1}$ and only $4 N$ real modes if $\Omega_{1}>\omega_{1}$.

Remark. Gyroscopic Quantum Oscillators. The instability results for the classical gyroscopic oscillator coupled to a heat bath can be extended directly to the quantum setting. This follows from the general quantization procedure for a classical system with quadratic Hamiltonian and gyroscopic terms.

The point is that the eigenvalue computation for the equations of motion in the classical setting is precisely equivalent to that for the Heisenberg equations of motion. Thus for the oscillator coupled to the heat bath the numerical computation in the classical case gives us the correct eigenvalue information for the quantum case also and we can deduce qualitative behavior as for the single gyroscopic oscillator in the previous section. Thus, for sufficiently large coupling, we observe unbound states even when the uncoupled gyroscopic oscillator exhibits bound states. 


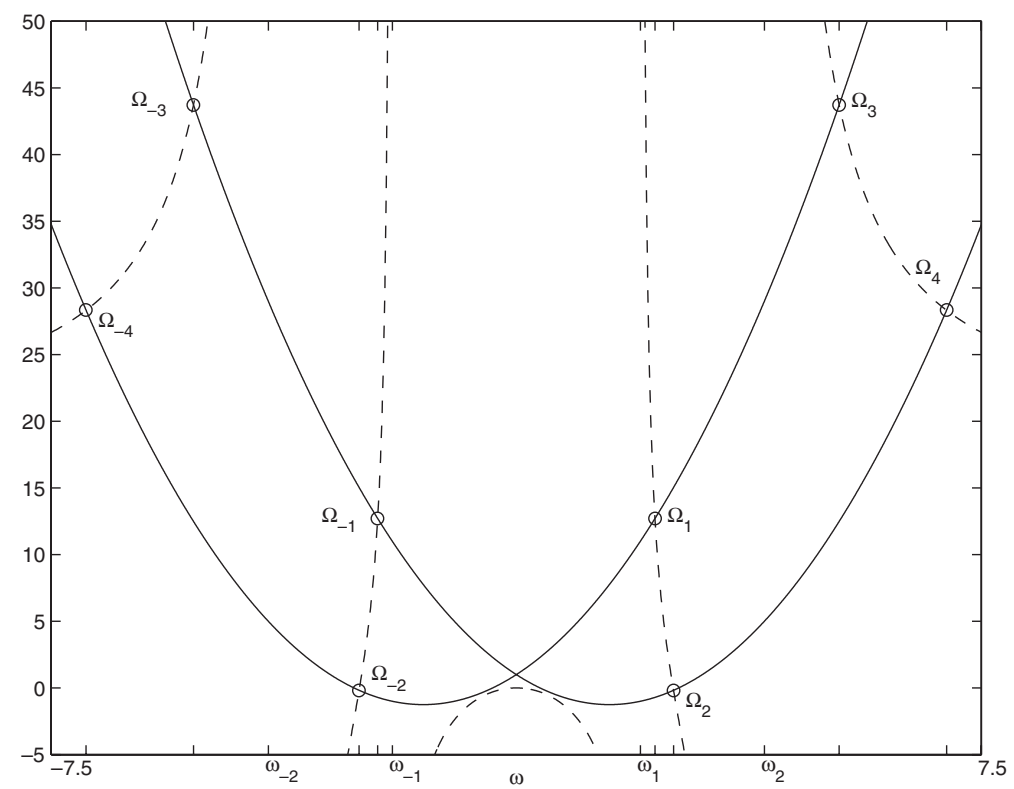

Fig. 6. Gyroscopic system destabilized via coupling to a heat bath of two oscillators $(N=2)$. Stable coupling with $\alpha=-1, B=3, m_{1}=1, \omega_{1}=1, m_{2}=1$, and $\omega_{2}=4$. Notice the system has only $4 N$ stable normal modes since $\Omega_{1}>\omega_{1}$. The dashed line is the graph of $\sum_{j=1}^{N} m_{j} \frac{\omega_{j}^{2} \omega^{2}}{\omega^{2}-\omega_{j}^{2}}$ while the solid lines are the graphs of the parabolas $\left(\omega \pm \frac{1}{2} B\right)^{2}-\left(\alpha+\frac{1}{4} B^{2}\right)$.

The quantization of the uncoupled gyroscopic system illustrates that the stability analysis of the quantum system is analogous to the classical normal mode calculation. However there are some subtleties in the interpretation of the stability analysis. We illustrate this briefly with a planar oscillator with gyroscopic forces:

We follow the Dirac formalism as described in refs. 26 and 25 for example.

In the case of a symmetric planar oscillator, we assume that $\alpha=\beta$ and the Hamiltonian reduces to $\left(\omega_{0}=\omega_{x_{0}}=\omega_{y_{0}}\right)$

$$
\begin{aligned}
H & =\hbar \omega_{0}\left(a_{0}^{\dagger} a_{0}+b_{0}^{\dagger} b_{0}+1\right)+\tilde{H}_{B}, \\
\tilde{H}_{B} & =i \frac{\hbar B}{2}\left(a_{0}^{\dagger} b_{0}-a_{0} b_{0}^{\dagger}\right) .
\end{aligned}
$$

By a standard change of coordinates we have a Hamiltonian in terms of normal modes: 


$$
\begin{aligned}
& A_{ \pm}=\frac{1}{\sqrt{2}}\left(a_{0} \mp i b_{0}\right) \\
& A_{ \pm}^{\dagger}=\frac{1}{\sqrt{2}}\left(a_{0}^{\dagger} \pm i b_{0}^{\dagger}\right) .
\end{aligned}
$$

The eigenvalues of the Hamiltonian, $E_{n_{1}, n_{2}}$ can be shown to be

$$
E_{n_{1}, n_{2}}=\hbar \omega_{0}\left(n_{1}+n_{2}+1\right)+\hbar \frac{B}{2}\left(n_{1}-n_{2}\right)
$$

where $n_{1}, n_{2} \in\{0,1,2, \ldots\}$. In the case of gyroscopic stability, we have $\frac{B}{2}>\omega_{0}$, and the energy spectrum is indefinite and unbounded above and below. In the case of pure stability $(\alpha>0)$, we have $\frac{B}{2}<\omega_{0}$, and the energy spectrum in positive definite.

In the case of an unstable gyroscopic oscillator, $\left(\frac{B}{2}+\min \{\alpha, \beta\}<0\right)$, there are no bound eigenstates.

Similary in the case of a quantum oscillator coupled to a finite heat bath we can reduce the coupled problem to a system of independent quantum oscillators. Thus the spectrum of the Hamiltonian of the coupled quantum system is positive if and only if the classical system is strongly stable; the spectrum is discrete with both postive and negative eigenvalues if and only if the classical system is gyroscopically stable; and finally, we have unbounded eigenfunctions if and only if the classical system in unstable.

\section{CONTINUUM COUPLING}

The above analysis was for a gyroscopic oscillator connected to a finite bath of discrete oscillators. It is of interest to investigate stability of the oscillator coupled to a continuum of oscillators and to see what new phenomena arise.

In the infinite setting dissipative effects, induced by coupling to the continuum bath, become important. Moreover, such dissipative effects induce instability as discussed for general gyroscopic systems in ref. 5. In our setting suppose we have a Rayleigh dissipation so that the gyroscopic oscillator equations become

$$
\begin{aligned}
& \ddot{x}-B \dot{y}+\alpha x+\gamma \dot{x}=0 \\
& \ddot{y}+B \dot{x}+\beta y+\gamma \dot{y}=0
\end{aligned}
$$


where $\gamma>0$. Then the normal mode equation (4.22) becomes

$$
\left(\omega \pm \frac{1}{2} B\right)^{2}-\left(\alpha+\frac{1}{4} B^{2}\right)=-i \gamma \omega
$$

or

$$
\omega^{2}+\omega(i \gamma \pm B)-\alpha=0
$$

which clearly has complex solutions in general and we obtain instability.

Now the natural limiting Lagrangian for our systems here is

$$
L=L_{0}+L_{\text {bath }}
$$

where

$$
L_{0}=\frac{1}{2}\left(\dot{x}^{2}+\dot{y}^{2}\right)-\frac{1}{2}\left(\alpha x^{2}+\beta y^{2}\right)+\frac{1}{2} B(x \dot{y}-y \dot{x})
$$

and

$$
L_{\text {bath }}=\int_{\mathbb{R}}\left(\frac{1}{2} m\left(\dot{u}^{2}+\dot{v}^{2}\right)-\frac{1}{2} m v^{2}\left((u-x)^{2}+(v-y)^{2}\right)\right) d v .
$$

Here $x(t)$ and $y(t)$ are the distinguished oscillator coordinates and $u(t, v)$, $v(t, v)$ denote continuum oscillator coordinates of natural frequency $v$. Here $m=m(v)$ is a mass density: $m(v) d v /\left(\int m\left(v^{\prime}\right) d v^{\prime}\right)$ denotes the proportion of oscillators with natural frequency between $v^{\prime}$ and $v^{\prime}+d v^{\prime}$.

The corresponding equations of motion are

$$
\begin{aligned}
\ddot{x}-B \dot{y}+\alpha x & =\int_{\mathbb{R}} m v^{2}(u-x) d v \\
\ddot{y}+B \dot{x}+\beta y & =\int_{\mathbb{R}} m v^{2}(v-y) d v \\
\int_{\mathbb{R}} m\left(\ddot{u}+v^{2}(u-x)\right) d v & =0 \\
\int_{\mathbb{R}} m\left(\ddot{v}+v^{2}(v-y)\right) d v & =0 .
\end{aligned}
$$

Assuming mass distributions with finite variance, the last two equation become

$$
\begin{aligned}
\ddot{u}+v^{2} u & =v^{2} x \\
\ddot{v}+v^{2} v & =v^{2} y .
\end{aligned}
$$


To obtain normal mode solutions, we set

$$
\begin{aligned}
x(t) & =X_{0}(\omega) e^{i \omega t} ; & y(t) & =Y_{0}(\omega) e^{i \omega t} \\
u(v, t) & =X_{v}(\omega) e^{i \omega t} ; & v(v, t) & =Y_{v}(\omega) e^{i \omega t} .
\end{aligned}
$$

The equations of motion for the normal modes become

$$
\begin{aligned}
& \left(-\omega^{2}+\alpha\right) X_{0}=-i B \omega Y_{0}+\int_{\mathbb{R}} m v^{2}\left(X_{v}-X_{0}\right) \\
& \left(-\omega^{2}+\beta\right) Y_{0}=i B \omega X_{0}+\int_{\mathbb{R}} m v^{2}\left(Y_{v}-Y_{0}\right) \\
& \left(-\omega^{2}+v^{2}\right) X_{v}=v^{2} X_{0} \\
& \left(-\omega^{2}+v^{2}\right) Y_{v}=v^{2} Y_{0} .
\end{aligned}
$$

Eliminating the bath coordinates, we have

$$
\left[\begin{array}{cc}
\omega^{2}-\alpha+\int_{\mathbb{R}} m \frac{v^{2} \omega^{2}}{v^{2}-\omega^{2}} d v & -i B \omega \\
i B \omega & \omega^{2}-\beta+\int_{\mathbb{R}} m \frac{v^{2} \omega^{2}}{v^{2}-\omega^{2}} d v
\end{array}\right]\left[\begin{array}{c}
X_{0} \\
Y_{0}
\end{array}\right]=\left[\begin{array}{l}
0 \\
0
\end{array}\right] .
$$

For nontrivial solution, we require

$$
\left(\omega^{2}-\alpha+\int_{\mathbb{R}} m \frac{v^{2} \omega^{2}}{v^{2}-\omega^{2}} d v\right)\left(\omega^{2}-\beta+\int_{\mathbb{R}} m \frac{v^{2} \omega^{2}}{v^{2}-\omega^{2}} d v\right)=B^{2} \omega^{2} .
$$

In the case where $\alpha=\beta$, we can simplify the dispersion relation,

$$
\left(\omega \pm \frac{1}{2} B\right)^{2}-\left(\alpha+\frac{1}{4} B^{2}\right)=\int_{\mathbb{R}} m(v) \frac{v^{2} \omega^{2}}{\omega^{2}-v^{2}} d v
$$

This dispersion relationship in the infinite-dimensional setting requires careful interpretation. As in the finite-dimensional setting the above analyis is simply an analysis of the normal modes of the system. Any complex frequencies will give to an instability. One way to interpret the integral in the dispersion relation is the principle value sense. We then see that the frequencies $\omega$ which satisfy the dispersion relation form a discrete set. If $m$ decays fast enough for the principle value of integral to be finite, we have only a finite number of real solutions. Increasing $m$ from zero, the corresponding solutions are the perturbed stable eigenvalues of the discrete 


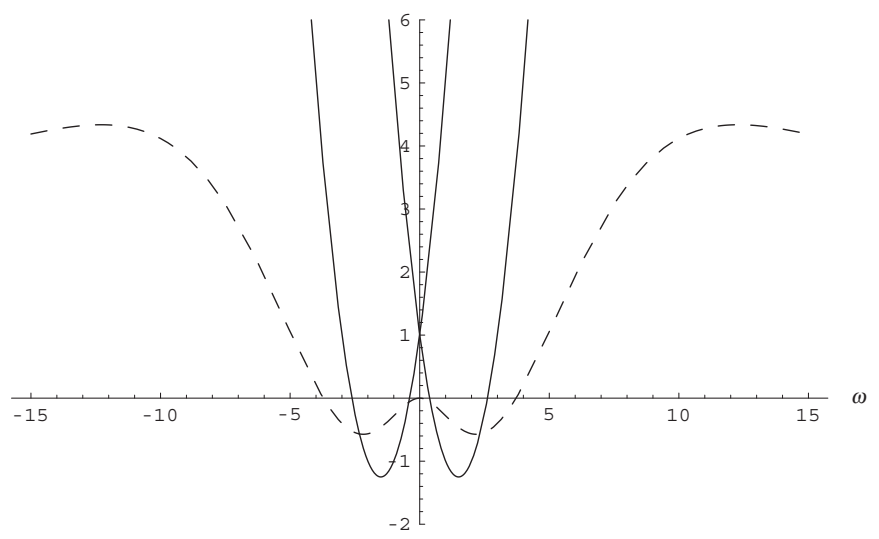

Fig. 7. Continuum coupling to gyroscopic system. "Stable" coupling with $\alpha=-1, B=3$, and $m(v)=\frac{1}{10} e^{-\frac{|v|}{2}}$. The dashed line is the graph of P.V. $\int_{\mathbb{R}} m \frac{v^{2} \omega^{2}}{\omega^{2}-v^{2}} d v$ while the solid lines are the graphs of the parabolas $\left(\omega \pm \frac{1}{2} B\right)^{2}-\left(\alpha+\frac{1}{4} B^{2}\right)$.

system. If there are 4 real modes, then we consider the oscillator to be stable. If there are fewer than 4 real modes, then the motion of the coupled oscillator must include unstable modes. In Fig. 7, we see that there exist enough stable modes to describe the motion of the oscillator. Increasing the mass of the bath can lower the number of real modes. For example, Fig. 8 has no real modes and is hence unstable.

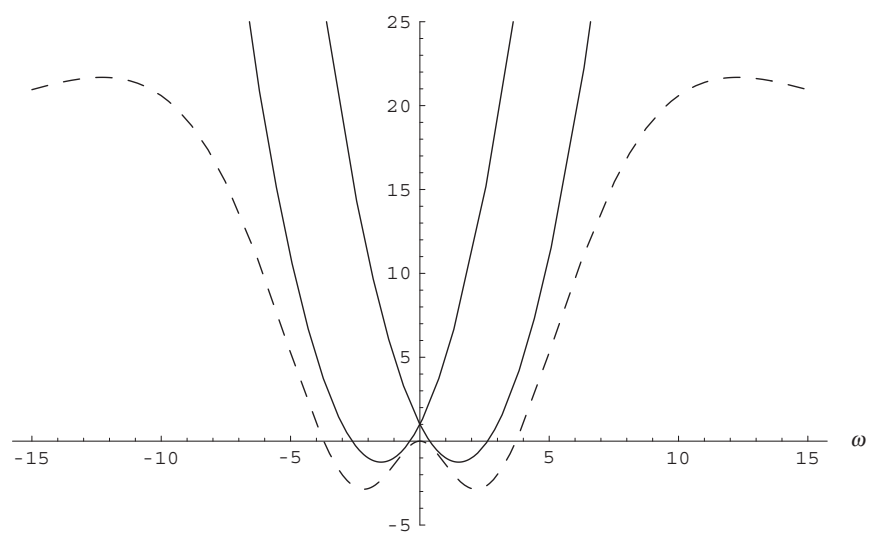

Fig. 8. Continuum coupling to gyroscopic system. "Unstable" coupling with $\alpha=-1, B=3$, and $m(v)=\frac{1}{2} e^{-\frac{|v|}{2}}$. The dashed line is the graph of P.V. $\int_{\mathbb{R}} m \frac{v^{2} \omega^{2}}{w^{2}-v^{2}} d v$ while the solid lines are the graphs of the parabolas $\left(\omega \pm \frac{1}{2} B\right)^{2}-\left(\alpha+\frac{1}{4} B^{2}\right)$. 
On the other hand in the infinite setting it is possible to get a further mechanism for instability - damping, as in the Rayleigh damped case discussed above. In the infinite setting one can see this in the analysis of the initial value problem, carried out for example by Laplace transform analysis. The Fourier mode analysis imposes a time reversal symmetry and cannot capture the damping revealed by a Laplace transform analysis as in the case of Landau damping of plasmas (see, e.g., refs. 21 and 28). In our setting the system is time reversible and each individual mode may be stable or unstable. However, as in the Landau setting, a superposition of infinitely many modes can damp as they drift out of phase, following the argument of ref. 17. This damping effect leads to instability in the gyroscopic setting even if the individual modes are stable.

We construct an instability as follows. We show that for $\epsilon>0$ and small there is a solution of

$$
\left(\omega \pm \frac{1}{2} B\right)^{2}-\left(\alpha+\frac{1}{4} B^{2}\right)-\epsilon \int_{\mathbb{R}} m(v) \frac{v^{2} \omega^{2}}{\omega^{2}-v^{2}} d v=0,
$$

$\omega=\omega(\epsilon)$ with negative imaginary part, $\Im \omega<0$ (so that $e^{i \omega t}$ grows as $t$ increases), and a corresponding finite energy normal mode (5.14).

Let

$$
F(\omega, \epsilon) \equiv\left(\omega+\frac{\tau_{1}}{2} B\right)^{2}-\left(\alpha+\frac{1}{4} B^{2}\right)-\epsilon \int_{C_{\delta}} m(v) \frac{v^{2} \omega^{2}}{\omega^{2}-v^{2}} d v=0,
$$

where $\tau_{1}= \pm 1$ and $C_{\delta}$ is a contour which coincides with the real axis, traversed from $-\infty$ to $+\infty$ for $|v| \geqslant \delta$ and which, for $|v|<\delta$, consists of a semicircular arc of radius $\delta$ in the upper half plane.

Begin with any one of the four roots, $\omega_{\tau_{1}, \tau_{2}}$, of the unperturbed problem, $\epsilon=0$ :

$$
\omega_{\tau_{1}, \tau_{2}}=\frac{\tau_{1}}{2} B+\frac{\tau_{2}}{2}\left(\alpha+\frac{1}{4} B^{2}\right)^{1 / 2}, \quad \tau_{1}, \tau_{2}= \pm 1 .
$$

Note that $F(\omega, \epsilon)$ is analytic for $\omega$ varying over the region below the contour $C_{\delta}$ and $\epsilon \in \mathbb{R}$. Furthermore, $F\left(\omega_{\tau_{1}, \tau_{2}}, 0\right)=0$. Using the implicit function theorem, we shall construct a solution of $F(\omega, \epsilon)=0$, with $\omega(\epsilon)$ near $\omega_{\tau_{1}, \tau_{2}}$ and $\mathfrak{I}(\omega(\epsilon))<0$ for $\epsilon$ near zero. Calculating, we find

$$
\begin{aligned}
F_{\omega}(\omega, \epsilon)= & 2\left(\omega+\frac{\tau_{1}}{2} B\right)+2 \epsilon \omega^{3} \int_{C_{\delta}} m(v) \frac{v^{2}}{\left(\omega^{2}-v^{2}\right)^{2}} d v \\
& -2 \epsilon \omega \int_{C_{\delta}} m(v) \frac{v^{2}}{\omega^{2}-v^{2}} d v
\end{aligned}
$$


and therefore

$$
F_{\omega}\left(\omega_{\tau_{1}, \tau_{2}}, 0\right)=\tau_{2}\left(\alpha+\frac{1}{4} B^{2}\right)^{\frac{1}{2}} \neq 0 .
$$

It follows that for $\epsilon$ sufficiently small, there is a function $\omega(\epsilon)$, satisfying $F(\omega(\epsilon), \epsilon)=0$. Differentiation gives:

$$
\begin{aligned}
\frac{d \omega}{d \epsilon}(0) & =-F_{\epsilon}\left(\omega_{\tau_{1}, \tau_{2}}, 0\right) F_{\omega}^{-1}\left(\omega_{\tau_{1}, \tau_{2}}, 0\right) \\
& =-\left(\tau_{2}\left(\alpha+\frac{1}{4} B^{2}\right)^{\frac{1}{2}}\right)^{-1} F_{\epsilon}\left(\omega_{\tau_{1}, \tau_{2}}, 0\right)
\end{aligned}
$$

and therefore the sign of the imaginary part of $\omega(\epsilon)$, for small $\epsilon$, is determined by the sign of

$$
F_{\epsilon}\left(\omega_{\tau_{1}, \tau_{2}}, 0\right)=-\omega_{\tau_{1}, \tau_{2}}^{2} \int_{C_{\delta}} m(v) \frac{v^{2}}{\omega_{\tau_{1}, \tau_{2}}^{2}-v^{2}} d v,
$$

provided it is nonzero. By analyticity of the integrand in (5.28), we can evaluate $F_{\epsilon}\left(\omega_{\tau_{1}, \tau_{2}}, 0\right)$ by taking letting $\delta$ tend to zero. We obtain

$$
F_{\epsilon}\left(\omega_{\tau_{1}, \tau_{2}}, 0\right)=-\omega_{\tau_{1}, \tau_{2}}^{2} \text { P.V. } \int_{\mathbb{R}} m(v) \frac{v^{2}}{\omega_{\tau_{1}, \tau_{2}}^{2}-v^{2}} d v+i \pi \omega_{\tau_{1}, \tau_{2}}^{3} m\left(\omega_{\tau_{1}, \tau_{2}}\right) .
$$

It follows that

$$
\mathfrak{I}\left(\frac{d \omega}{d \epsilon}(0)\right)=-\left(\tau_{2}\left(\alpha+\frac{1}{4} B^{2}\right)^{\frac{1}{2}}\right)^{-1} \pi \omega_{\tau_{1}, \tau_{2}}^{3} m\left(\omega_{\tau_{1}, \tau_{2}}\right),
$$

which can be made negative for appropriate choice of $\tau_{1}$ and $\tau_{2}$. And therefore, there exists a solution of $F(\omega(\epsilon), \epsilon)=0$ for $\epsilon$ small with $\mathfrak{I}(\omega(\epsilon))<0$. Finally, since $\mathfrak{I}(\omega(\epsilon))<0$ we may deform the contour $C_{\delta}$ in the definition of $F(\omega, \epsilon)$ back to the real axis and we have that $\omega(\epsilon)$ actually solves the desired Eq. (5.22). The corresponding normal mode (5.14) can be constructed algebraically and for mass-densities, $m(v)$, which decay sufficiently rapidly as $v$ tends to infinity, is easily seen to have finite energy. Thus as in the case of Rayleigh dissipation there is a complex root generically which leads to instability.

\section{RELATIONSHIP TO OTHER MODELS AND FINAL REMARKS}

In this final section we discuss other models of coupling to a thermal reservoir and their relationship to the stability analysis we have conducted in this paper. 
One may for example consider an oscillator connected to an infinite heat bath in the form of a string. We dicuss this briefly here. Further details may be found in refs. 12 and 13 .

One such model is based on the Lamb model ${ }^{(20)}$ of an oscillator is physically coupled to a string. The vibrations of the oscillator transmit waves into the string and are carried off to infinity. Hence, the oscillator loses energy and is effectively damped by the string.

Let $w(x, t)$ be the displacement of the string at position $x \in \mathbb{R}$ at time $t$. Let $\rho$ be the mass density of the string and let $T$ be the tension. We can compute the wave speed of transverse oscillations of the free string to be $c=\frac{T}{\rho}$. Assuming a singular mass density at $x=0$, we can couple the dynamics of an oscillator, $q$, of mass $M$, to the otherwise free wave to resolve the singularity,

$$
\begin{aligned}
\frac{\partial^{2} w}{\partial t^{2}} & =c^{2} \frac{\partial^{2} w}{\partial x^{2}} \\
M \ddot{q}+V q & =T\left[w_{x}\right]_{x=0} \\
q(t) & =w(0, t),
\end{aligned}
$$

where $\sqrt{\frac{V}{M}}$ is the frequency of the uncoupled oscillating mass $M$ and $\left[w_{x}\right]_{x=0}=w_{x}(0+, t)-w_{x}(0-, t)$ is the jump discontinuity of the slope of the string.

Perturbing the oscillator at time $t=0$ from its equilibrium position, we can use d'Alembert's solution to the wave equation to solve for $w$,

$$
w=\left\{\begin{array}{lll}
C e^{(c t-|x|) \omega} & \text { for } & |x|<c t, \\
0 & \text { for } & |x|>c t,
\end{array}\right.
$$

where $\omega=-\frac{T}{M c}+i \sqrt{\frac{V^{2}}{M^{2}}-\left(\frac{T}{M c}\right)^{2}}$ and $C$ is the size of the initial displacement. For small tension we have damping and oscillator motion, while for large tension, we have pure damping. From the solution of the wave equation, we can compute the jump condition of the oscillator,

$$
\left[w_{x}\right]_{x=0}=-2 C \omega e^{\omega c t}=-\frac{2}{c} \dot{q} .
$$

We obtain a reduced form of the dynamics describing the explicit motion of the oscillator subsystem,

$$
M \ddot{q}+\frac{2 T}{c} \dot{q}+V q=0 .
$$


The coupling term arises explicitly as a Rayleigh dissipation term $\frac{2 T}{c} \dot{q}$ in the dynamics of the oscillator. For the gyroscopic Lamb coupling, the dissipation term will induce instabilities in gyroscopically stabilized Chetaev systems.

A variant of the Lamb model, is a system which includes gyroscopic terms coupled to the standard wave equation. We have the boundary constraint that the displacement of the gyroscopic oscillator fixes a point of the string. One can show that this local coupling perturbation destabilizes gyroscopically stabilized systems.

We consider a model of a string in $\mathbb{R}^{n+1}$, whose transverse vibrations are independent. Suppose the string lies initially along the $x_{n+1}$-axis. For simplicity, we denote the $x_{n+1}$ dimension as the $z$ dimension. Coupling the transverse motion to a $n$-dimensional gyroscopic system, we can solve the system

$$
\begin{aligned}
\frac{\partial^{2} \mathbf{w}}{\partial t^{2}}(z, t) & =c^{2} \frac{\partial^{2} \mathbf{w}}{\partial z^{2}}(z, t), \quad z \in \mathbb{R}-\{0\}, \quad t \in \mathbb{R}, \\
M \ddot{\mathbf{q}}(t)+S \dot{\mathbf{q}}(t)+V \mathbf{q}(t) & =T\left[\frac{\partial \mathbf{w}}{\partial z}\right]_{z=0} \\
\mathbf{w}(0, t) & =\mathbf{q}(t),
\end{aligned}
$$

where $c$ is the speed of transverse waves in the string, $T$ is the tension of the string, $\mathbf{w}=\left[w_{1}(z, t) \cdots w_{n}(z, t)\right]^{T}$ is the displacement of the string in the first $n$ dimensions and $\left[\frac{\partial \mathrm{w}}{\partial z}\right]_{z=0}$ is the jump discontinuity in the slope of the string. Let us define the initial conditions on the string by $\mathbf{w}_{1}(z)=\frac{\partial \mathrm{w}}{\partial t}(z, 0)$, and $\mathbf{w}_{0}(z)=\mathbf{w}(z, 0)$.

By direct computation we obtain an exact reduced dynamical system for q. This reduction contains explicit dissipation terms reflecting the oscillator/field coupling. The results on reduction and stability are summarized in the following two results.

Proposition 6.1. If the initial data $\mathbf{w}_{0}$ and $\mathbf{w}_{1}$ have compact support, then for $\pm c t \notin \operatorname{supp}\left(\mathbf{w}_{0}\right) \cup \operatorname{supp}\left(\mathbf{w}_{1}\right)$ the oscillator dynamics of the gyroscopic Lamb model reduces to

$$
M \ddot{\mathbf{q}}(t)+\left(S+\frac{2 T}{c}\right) \dot{\mathbf{q}}(t)+V \mathbf{q}(t)=0
$$


A proof of this proposition may be found in ref. 13. In ref. 5, small dissipation is shown to induce instability in gyroscopically stable systems (see Theorem 3.2). In the gyroscopic Lamb model, radiation (energy transfer into an infinite-dimensional system) produces a Rayleigh dissipation term which depends on the tension of the string. Since dissipation induces instability in these system for small dissipation, small tension coupling yields radiation induced instability via an analogous mechanism. We summarize the main result of radiation induced instability in gyroscopically stabilized Chetaev systems with a theorem.

Theorem 6.2. If a gyroscopic mechanical system is gyroscopically stable (i.e., $V$ has a negative eigendirection), then local coupling via the Lamb model induces instability for small coupling parameter $T$.

A related model is a field coupling of the mechanical gyroscopic system to the wave equation.

We model force of the wave field on the Chetaev system by the magnitude of the wave field. Coupling of this type is important in various physical models - see refs. 29-31, and references therein. The interaction between the wave equation and the gyroscopic system is modeled with a coupling parameter $\kappa$ and with a coupling distribution $\chi(\xi)$; the equations of motion are

$$
\begin{aligned}
& M \ddot{\mathbf{q}}+S \dot{\mathbf{q}}+V \mathbf{q}=\kappa \int_{\mathbb{R}} \chi(z) w(z, t) d z\left[\begin{array}{c}
1 \\
\vdots \\
1
\end{array}\right], \\
& \frac{\partial^{2} w}{\partial t^{2}}-c^{2} \frac{\partial^{2} w}{\partial z^{2}}=\kappa \chi(z)\left[\begin{array}{c}
1 \\
\vdots \\
1
\end{array}\right]^{T} \mathbf{q} .
\end{aligned}
$$

Again one can show that instability is induced.

Other models of field coupling are also possible and of interest depending on the physical situation of interest. We mention two further models (ignoring gyroscopic forces):

One model is analyzed in ref. 33 who consider a Lagrangian for an oscillator connected to a free field as follows:

$$
L_{1}=\int\left\{\left[\dot{\phi}^{2}-\left(\frac{\partial \phi}{\partial x}\right)^{2}\right]+\delta(x)\left(\dot{q}^{2}-\Omega_{0}^{2} q^{2}-\epsilon q \frac{\partial \phi}{\partial t}\right)\right\} d t d x
$$


The equations of motion are (taking the mass to be unity)

$$
\begin{aligned}
& \left(\ddot{q}+\Omega_{0}^{2}\right) q=-\epsilon \frac{\partial \phi}{\partial t}(0, t) \\
& \frac{\partial^{2} \phi}{\partial t^{2}}-\frac{\partial^{2} \phi}{\partial x^{2}}=\epsilon \dot{q} \delta(x) .
\end{aligned}
$$

It is easy to see how damping arises in the model (6.9) and furthermore, see how the system decouples (see ref. 3 for related details):

Taking the Fourier transform with respect to $x$ in the wave equation yields

$$
\begin{aligned}
\hat{\phi}(k, t) & =\int_{\mathbb{R}} e^{-i k x} \phi(x) d x, \\
\hat{\phi}_{t t}(k, t)+k^{2} \hat{\phi}(k, t) & =\epsilon \dot{q}(t) .
\end{aligned}
$$

Solving the o.d.e. for $\hat{\phi}$, we have

$$
\hat{\phi}(k, t)=\epsilon \int_{0}^{t} \frac{\sin (k(t-s))}{k} q(s) d s+\hat{\phi}_{\mathrm{free}}(k, t) .
$$

The above equation is the retarded Green's function for the wave equation and $\phi_{\text {free }}$ is the homogeneous solution satisfying the initial conditions. Neglecting $\phi_{\text {free}}$, we have

$$
\begin{aligned}
\phi(0, t) & =\frac{\epsilon}{2} \int_{0}^{t} \operatorname{sgn}(t-s) \dot{q}(s) d s \\
& =\frac{\epsilon}{2} \int_{0}^{t} \dot{q}(s) d s .
\end{aligned}
$$

Thus we obtain

$$
\ddot{q}+\Omega_{0}^{2} q=-\frac{\epsilon^{2}}{2} \dot{q}
$$

If the free term is included one obtains an additional forcing term of $-\epsilon \dot{\phi}_{\text {free }}$ on the right hand side.

Finally it is of interest to consider the model in ref. 19. 
In this case one has a scalar wave field $\phi(x)$ in three dimensions coupled to a particle with position $q$. Ignoring relativistic effects the Lagrangian is of the form

$$
L_{2}=\frac{1}{2} \dot{q}^{2}+\frac{1}{2} \int d^{3} x\left(\left(\frac{\partial \phi(x, t)}{\partial t}\right)^{2}-|\nabla \phi(x, t)|^{2}\right)+\int d^{3} x \phi(x, t) \rho(x-q) .
$$

In ref. $19 \rho(x)$ is chosen to ensure the energy is bounded from below. We note also the difference between this model and the model (6.7). Here the model is that of oscillator coupled to a scalar field in three dimensions while the model (6.7) is that of an oscillator system coupled via a coupling parameter to $n$ copies of a scalar field of one dimension. This latter model is of interest for analysis of resonances in quantum systems (see ref. 31) while the model of ref. 19 is of interest for certain physical models of a particle interacting with a field.

\section{ACKNOWLEDGMENTS}

We would like to thank the referees for their helpful comments and suggestions and Peter Smereka for useful discussions. The research of A.M.B. was partially supported by National Science Foundation Grants DMS 0103895 and 0305837 . The research of P.H. was partially supported by National Science Foundation and AFOSR. The research of M.I.W. was partially supported by the National Science Foundation.

\section{REFERENCES}

1. V. Bach, J. Fröhlich, and I. Sigal, Return to equilibrium, J. Math. Phys. 41:3985-4060 (2000).

2. J. Baillieul and M. Levi, Constrained relative motions in rotational mechanics, Arch. Rational Mech. Anal. 115:101-135 (1991).

3. A. M. Bloch, Dissipation dynamics in classical and quantum conservative systems, in Mathematical Systems Theory in Biology, Communications, Computation, and Finance, J. Rosenthal and D. Gilliam, eds., (IMA, Springer Verlag, 2003), 121-156.

4. A. M. Bloch, P. J. Hagerty, A. G. Rojo, and M. Weinstein, Classical and quantum gyroscopic instability and heat baths, in Proc. 40th IEEE Conference on Decision and Control (2001).

5. A. M. Bloch, P. S. Krishnaprasad, J. E. Marsden, and T. S. Ratiu, Dissipation induced instabilities, Ann. Inst. H. Poincaré Anal. Non Linéaire 11:37-90 (1994).

6. L. S. Brown and G. Gabrielse, Geonium theory: Physics of a single electron or ion in a Penning trap, Rev. Modern Phys. 58:233-310 (1986).

7. A. Caldeira and A. Leggett, Path integral approach to quantum Brownian motion, Physica A 121:587-616 (1983).

8. N. G. Chetaev, The Stability of Motion (Pergamon Press, New York, 1961). 
9. J. Derezinski and V. Jaksic, Return to equilibrium for Pauli-Fierz systems, to appear in Ann. H. Poincaré (2002).

10. J. Derezinski, V. Jaksic, and C.-A. Pillet, Perturbation theory of $\mathrm{W}^{*}$-dynamics, Liouvilleans, and KMS-states, Rev. Math. Phys. 15:447-489 (2003).

11. G. W. Ford, J. T. Lewis, and R. F. O'Connell, Independent oscillator model of a heat bath: Exact diagonalization of the Hamiltonian, J. Stat. Phys. 53:439-455 (1988).

12. P. J. Hagerty, A. M. Bloch, and M. I. Weinstein, Radiation induced instability in interconnected systems, in Proc. 38th IEEE Conference on Decision and Control (1999).

13. P. J. Hagerty, A. M. Bloch, and M. I. Weinstein, Radiation induced instability, to appear in SIAM J. Appl. Math. (2003).

14. W. Hahn, Stability of Motion (Springer-Verlag, New York, 1967).

15. V. Jaksic and C.-A. Pillet, On a model for quantum friction I: Fermi's golden rule and dynamics at zero temperature, Ann. Inst. H. Poincaré Phys. Théor. 62:47-68 (1995).

16. V. Jaksic and C.-A. Pillet, Ergodic properties of the non-Markovian Langevin equation, Lett. Math. Phys. 41:1757-1780 (1997).

17. N. Kampen and B. U. Felderhof, Theoretical Methods in Plasma Physics (North-Holland, Amsterdam, 1967).

18. E. Kirr and M. I. Weinstein, Parametrically excited Hamiltonian partial differential equations, Siam J. Math. Anal. 33:16-52 (2001).

19. A. Komech, M. Kunze, and H. Spohn, Effective dynamics for a mechanical particle coupled to a wave field, Comm. Math. Phys. 203:1-19 (1999).

20. H. Lamb, On the peculiarity of the wave-system due to the free vibrations of a nucleus in an extended medium, Proc. London Math. Soc. 32:208-211 (1900).

21. L. Landau, On the vibrations of the electronic plasma, J. Phys. (USSR) 10:25 (1946).

22. J. Lebowitz, Microscopic reversibility and macrosopic behavior: Physical explanations and mathematical derivations, in Lecture Notes in Physics, J. J. Brey et al., eds. (SpringerVerlag, 1994).

23. J. E. Marsden and T. S. Ratiu, Introduction to Mechanics and Symmetry (Springer-Verlag, New York, 1999).

24. M. Merkli and I. Sigal, On time-dependent theory of quantum resonances, Comm. Math. Phys. 201:549-576 (1999).

25. A. Messiah, Quantenmechanik, Band 2 (de Gruyter, Berlin, 1990).

26. A. Messiah, Quantenmechanik, Band 1 (de Gruyter, Berlin, 1991).

27. J. C. Simo, D. Lewis, and J. E. Marsden, Stability of relative equilibria. I. The reduced energy-momentum method, Arch. Rational Mech. Anal. 115:15-59 (1991).

28. P. Smereka, Synchronization and relaxation for a class of globally coupled oscillator systems, Physica D 124:104-125 (1998).

29. A. Soffer and M. I. Weinstein, Nonautonomous Hamiltonians, J. Stat. Phys. 93:359-391 (1998).

30. A. Soffer and M. I. Weinstein, Time dependent resonance theory, Geom. Funct. Anal. 8:1086-1128 (1998).

31. A. Soffer and M. I. Weinstein, Resonances, radiation damping and instability in Hamiltonian nonlinear wave equations, Invent. Math. 136:9-74 (1999).

32. L. Thomson and P. G. Tait, Principles of Mechanics and Dynamics (Cambridge University Press, 1897).

33. W. Unruh and W. Zurek, Reduction of a wave packet in quantum Brownian motion, Phys. Rev. 400:1071-1094 (1989). 